\title{
Correspondence
}

\begin{abstract}
Allergic bronchopulmonary disease caused by fungi other than Aspergillus
\end{abstract}

Sir,-Dr McAleer and colleagues (Thorax 1981;36:338) report allergic bronchopulmonary disease related to fungal species other than aspergillus. We have identified two similar cases in an equivalent climatic region.

Case 1 A 29-year-old man with a history of childhood asthma was found to have a persisting asymptomatic right upper lobe opacity in a mass miniature $x$-ray survey in 1972. Radiographic appearances were consistent with mucoid impaction. Blood eosinophils were 2300 per $\mathrm{cm}$. Aspergillus prick tests were negative. A bronchial cast and viscus mucus were removed from the right upper lobe at bronchoscopy. These contained numerous fungal elements. Culture of these specimens grew Helminthorporum $s p$ only.

Case 2 A 28-year-old man with a history of childhood asthma was found to have a persisting asymptomatic right upper lobe opacity on mass miniature x-ray survey in 1976. The appearances suggested mucoid impaction. Blood eosinophils were $752 \mathrm{~cm}$. Precipitins against aspergillus were negative. Thick viscid mucus removed from the right upper bronchus at bronchoscopy contained many mycelial elements. Culture grew only Curvalaria sp.

The cultures in both cases were confirmed at the School of Tropical Health, University of Sydney. The patients lived at latitude $19^{\circ} \mathrm{S}$ on the eastern seaboard of Australia. The latitude and climate are equivalent to the regions where Dr McAleer's cases originated, but on the other side of the continent.

In a 10-year period from 1971 in this region approximately 10-20 new cases of mucoid impaction caused by Aspergillus $s p$ have been identified. Our observations, together with those of Dr McAleer and colleagues, suggest that these other types of organism are also a significant cause of allergic bronchopulmonary disease across the tropical zone of Australia.

AM MATTHIESSON

Chest Clinic

General Hospital

Townsville

Queensland

Australia

\section{Oral VP-16-213 in advanced bronchogenic carcinoma}

Sir,-The report (Anderson et al. Thorax, 1981;35:462.) of oral VP-16-213 in advanced bronchogenic carcinoma demonstrates clinical activity of this agent. The authors found unexplained marrow toxicity in three pilot studies combining oral VP-16-213 with vincristine and varying doses of methotrexate and with vincristine and a small dose of adriamycin. They postulate this might have been the result of combination of VP-16-213 with methotrexate but this is difficult to understand as one of the schedules did not contain methotrexate. Since vincristine was common to all three schedules and is frequently used in combination chemotherapy for bronchogenic carcinoma, it is important to consider other possibilities.

The exact cell types and extent of disease particularly affecting bone or bone marrow were not reported in the pilot studies, and it is possible the severe pancytopenia reported might have been caused by unrecognised marrow involvement by widely disseminated small cell lung cancer, unresponsive to cytotoxic drug therapy. The already compromised marrow may have been further depressed by even one course of cytotoxic chemotherapy.

An alternate explanation might be drug interaction. VP-16-213 at typical plasma concentrations is $94 \%$ bound to human serum albumin (Arnold. Cancer Chemother Pharmacol, 1979;3:71.). Vincristine has been reported to bind to formed blood elements (Owellen et al. Cancer Res, $1977 ; 37: 2603$.). It is possible the normally nonmyelosuppressive vincristine might displace VP-16-213 from its plasma binding sites and by this mechanism increase VP-16-213 myelosuppression. Since, in future studies, VP-16-213 is likely to be combined with other drugs in the treatment of lung cancer, it is important that drug interactions be elucidated.

B CANTWELL Department of Medical Oncology, St Vincent's Hospital, Dublin

Sir,-We postulated an interaction between VP-16-213 and methotrexate because there were two theoretical reasons for such an interaction. We specifically stated "neither theory explains marrow depression with Schedule C which did not contain methotrexate". Vincristine does not seem responsible because we have subsequently found marrow toxicity with VP-16-213 200 $\mathrm{mg}$ orally daily for five days combined with cyclophosphamide $500 \mathrm{mg}$ intravenously on days 1 and 2 . The common feature in all four marrow-toxic combinations of VP-16-213 with other cytotoxics is that VP-16-213 was given as $200 \mathrm{mg}$ orally for five days. A possible explanation has been suggested (Dr LA Price, personal communication). The administration of a combination of drugs on days 1 and 2 kills cycling marrow stem cells, thus drawing stem cells from $\mathrm{G}_{0}$ into cycle. The relatively prolonged duration of giving VP-16-213 over five days allows these recently introduced marrow stem cells to be killed by this drug causing pancytopenia. If this explanation is correct then the same total dose of 1 gramme of VP-16-213 given over two days should avoid marrow 
depression and this is being studied.

To answer Dr Cantwell's other points. The extent of disease is shown in table 1 of our paper. Routine marrow biopsies were not done because it would be cruel and even if done and negative would not exclude marrow metastases at other sites. It is difficult to see how undiagnosed marrow metastases would fail to cause marrow depression after multiple cycles of monoagent VP-16-213 yet would do so after a single cycle of the same drug when combined with modest doses of other cytotoxics.

GERALD ANDERSON Newport Chest Clinic Newport Gwent

\title{
Guy Scadding Travelling Fellowship in Respiratory Medicine
}

\begin{abstract}
Applications are invited for this Fellowship from medical practitioners, including consultants of not more than five years standing. Approximately $£ 500$ is available to support travel to medical centres, for studies related to respiratory medicine. Preference will be given to applicants proposing visits to centres in Europe. Applications should be sent to
\end{abstract}

Kenneth M Citron, MD, FRCP, Consultant Physician, Brompton Hospital, Fulham Road, London SW3 6HP 